ARTICLE

Received 5 May 2015 | Accepted 18 Dec 2015 | Published 1 Feb $2016 \quad$ DOl: 10.1038/ncomms10513 OPEN

\title{
Phylogenomic and biogeographic reconstruction of the Trichinella complex
}

Pasi K. Korhonen ${ }^{1, \star}$, Edoardo Pozio ${ }^{2, \star}$, Giuseppe La Rosa ${ }^{2, \star}$, Bill C.H. Chang ${ }^{1,3}$, Anson V. Koehler ${ }^{1}$, Eric P. Hoberg ${ }^{4}$, Peter R. Boag ${ }^{5}$, Patrick Tan ${ }^{6,7}$, Aaron R. Jex ${ }^{1}$, Andreas Hofmann ${ }^{1,8}$, Paul W. Sternberg ${ }^{9}$, Neil D. Young ${ }^{1}$ \& Robin B. Gasser ${ }^{1}$

Trichinellosis is a globally important food-borne parasitic disease of humans caused by roundworms of the Trichinella complex. Extensive biological diversity is reflected in substantial ecological and genetic variability within and among Trichinella taxa, and major controversy surrounds the systematics of this complex. Here we report the sequencing and assembly of 16 draft genomes representing all 12 recognized Trichinella species and genotypes, define protein-coding gene sets and assess genetic differences among these taxa. Using thousands of shared single-copy orthologous gene sequences, we fully reconstruct, for the first time, a phylogeny and biogeography for the Trichinella complex, and show that encapsulated and non-encapsulated Trichinella taxa diverged from their most recent common ancestor $\sim 21$ million years ago (mya), with taxon diversifications commencing $\sim 10-7$ mya.

\footnotetext{
${ }^{1}$ Faculty of Veterinary and Agricultural Sciences, The University of Melbourne, Melbourne, Victoria 3010, Australia. ${ }^{2}$ Istituto Superiore di Sanità, Viale Regina Elena 299, 00161 Rome, Italy. ${ }^{3}$ Yourgene Bioscience, Shu-Lin District, New Taipei City 23863, Taiwan. ${ }^{4}$ United States National Parasite Collection, US Department of Agriculture, Agricultural Research Service, Beltsville, Maryland 20705, USA. ${ }^{5}$ Department of Biochemistry and Molecular Biology, Monash University, Melbourne, Victoria 3800, Australia. ${ }^{6}$ Genome Institute of Singapore, 60 Biopolis Street, Singapore 138672 , Republic of Singapore. ${ }^{7}$ Cancer and Stem Cell Biology, Duke-NUS Graduate Medical School, Singapore 138672, Republic of Singapore. ${ }^{8}$ Structural Chemistry Program, Eskitis Institute, Griffith University, Brisbane, Queensland 4111, Australia. ${ }^{9}$ Division of Biology, Howard Hughes Medical Institute, California Institute of Technology, Pasadena, California 91125, USA. * These authors contributed equally to this work. Correspondence and requests for materials should be addressed to R.B.G.

(email: robinbg@unimelb.edu.au).
} 
$\mathrm{P}$ arasitic diseases cause substantial morbidity and mortality in billions of animals and humans worldwide, and also major losses to the global food production annually. Parasitic roundworms (nematodes) of the genus Trichinella cause disease (trichinellosis) in humans, which can lead to serious morbidity and mortality ${ }^{1,2}$. Although trichinellosis is endemic in many regions of the world, the predominant impact of human disease relates principally to acute outbreaks following consumption of infected, raw meat products ${ }^{2}$, with examples in Argentina, China, Laos, Papua New Guinea, Romania and Vietnam $^{3-9}$.

Trichinella is a complex of at least 12 species and genotypes, with a broad geographic range, including Europe, Africa, Asia, the Americas and Australasia ${ }^{10,11}$. Although only morphologically distinguishable groups (that is, encapsulated and nonencapsulated clades) of Trichinella are recognized, based on the appearance of larvae in muscle cells in infected hosts, molecular studies have defined nine species and three genotypes that display extensive biological diversity ${ }^{10-13}$. Currently, based on genetic data, the encapsulated clade (infecting only mammals) includes T. spiralis, T1; T. nativa, T2; T. britovi, T3; T. murrelli, T5; T. nelsoni, T7; T. patagoniensis, T12; and Trichinella genotypes T6, T8 and T9; and the non-encapsulated clade includes T. pseudospiralis, T4 (infecting mammals and birds); T. papuae, T10; and T. zimbabwensis, T11 (infecting mammals and reptiles). These taxa quite often represent localized populations, and exhibit varying degrees of intraspecific genetic variability (T. pseudospiralis), dispersal ability and host usage $\mathrm{e}^{12}$.

The biological diversity of Trichinella is of major evolutionary significance and reflects substantial genetic diversity, divergent ecology and host-parasite affiliations ${ }^{11,14,15}$. Despite some advances, there have been significant knowledge gaps in the biogeography and phylogeny of the Trichinella complex, as most previous studies represent analyses of small-scale molecular data sets, limiting interpretation and conclusions ${ }^{1-7}$. Progress in genomic, transcriptomic and bioinformatic technologies ${ }^{16-18}$ now provides unique opportunities to rapidly overcome such limitations and to enable research on Trichinella and trichinellosis. Although the nuclear genome of T. spiralis ${ }^{19}$ and the mitochondrial genomes of most Trichinella taxa have been characterized $^{20,21}$, there have been no global, nuclear genomic or transcriptomic data sets for the 11 other recognized taxa of Trichinella. In the present study, we sequenced and assembled draft genomes and transcriptomes for all currently recognized Trichinella species and genotypes and defined protein-coding gene sets. Using these data sets, we then reconstructed the phylogeny and biogeography of Trichinella, as a basis to address some pertinent and outstanding questions regarding the evolution and biology of Trichinella.

\section{Results}

Genomes and transcriptomes. We sequenced and assembled 16 draft genomes and 15 transcriptomes from all 12 currently recognized Trichinella taxa, including five distinct geographic isolates of T. pseudospiralis (Tables 1 and 2 and Supplementary Tables 1-4). The draft assemblies of these genomes (available publicly; see 'Accession codes' section) ranged from 46.1 to $51.5 \mathrm{Mb}$ (mean: $49.0 \mathrm{Mb}$ ), with average scaffold $\mathrm{N} 50 \mathrm{~s}$ of $106-294 \mathrm{~kb}$ (mean: $196 \mathrm{~kb}$ ) and GC contents of $32.5-33.7 \%$ (33.2\%; Tables 1 and 2). For all assemblies, 96.4\% (95.6-97.2\%) of all 248 core essential genes were identified (Tables 1 and 2), indicating that the gene sets represent substantial proportions of individual genomes. The repeat contents of individual draft genomes were estimated at $6.7-21.8 \%$ (mean: $17.7 \%$ ) of their total nucleotide compositions (Supplementary Tables 5 and 6). On average, the assemblies contain $2.4 \%$ (range: 1.1-3.7\%) retrotransposons, $2.9 \%(0.7-5.5 \%)$ DNA transposons, $7.7 \%$ (0.04-10.6\%) unclassified dispersed elements and $4.8 \%$ (4.1-5.8\%) simple repeats (Supplementary Tables 5 and 6). The repeat content of the genome of genotype T9 $(6.7 \%)$ is exceptionally low compared with those of all other Trichinella taxa (mean: 17.7\%). A comparison of the present draft genome for T. spiralis (ISS3) with that published previously for same species (ISS195) ${ }^{19}$ revealed essentially the same percentage of core essential genes (96\%), and repeat and GC contents, but a size difference $(14 \mathrm{Mb})$, likely to relate to differing sequencing and assembly methodologies or a genetic distinctiveness between the two geographic isolates.

Protein-encoding gene sets. From the 16 genomes representing all Trichinella taxa, we predicted 11,006-16,067 (mean: 13,912) protein-encoding genes that were $2,071-3,169 \mathrm{bp}$ in length (mean: 2,632; with introns), exons that were 170-223 bp in length (mean: 203) and introns that were 218-284 bp in length (mean: 259), with 5.7-6.9 exons per gene using complementary, de novo and homology-based approaches (Tables 1 and 2). These genes and introns are shorter than those of Trichuris suis (mean: 3,812 and $510 \mathrm{bp})$, Haemonchus contortus (6,167 and $832 \mathrm{bp}$ ), Ascaris suum (mean: 6,636 and 1,081 bp) and Toxocara canis $(8,416$ and $1,133 \mathrm{bp})$, but are similar to those of Brugia malayi $(3,106$ and $311 \mathrm{bp})$ and Caenorhabditis elegans $(3,680 \text { and } 320 \mathrm{bp})^{22-28}$. In total, 7,659-11,931 (mean: 9,697; 69.9\%) of the protein-encoding genes had homologous sequences (BLASTp $E$ value: $10^{-5}$ ) in the NCBI nr protein database (7,655-11,930; mean 9,694; 69.7\%), UniProtKB/SwissProt database (5,143-5,912; mean: 5,603; 40.3\%) and WormBase $(4,891-5,415$; mean: 5,$205 ; 37.4 \%)$. Genes with domain and motif matches in InterProScan $(n=5,289-6,372$; mean: 5,$847 ; 42.0 \%)$ contained $4,626-5,362(5,035 ; 36.2 \%)$ hits in Pfam, 5,018-5,879 (5,489; 39.5\%) in PANTHER, 929-1,037 (992; 7.1\%) in PRINTS and 234-266 (252; 1.8\%) in PIRSF databases (Supplementary Tables 7 and 8). In total, 7,691-12,000 (mean: 9,730) orthologous genes $\left(E\right.$ value $\left.\leq 10^{-5}\right)$ were associated with biological (KEGG) pathways. The secretomes of individual Trichinella taxa were predicted to comprise 314-414 (mean: 363) excretory/secretory (ES) proteins, 238 of which are encoded by single-copy orthologous genes (SCOs; Supplementary Data 1) and include cysteine, metallo- and serine proteases, peptidase inhibitors and orphan (uncharacterized) proteins identified previously ${ }^{29-32}$.

Phylogeny. We reconstructed the phylogeny of all 12 currently recognized Trichinella taxa ( $c f$. Tables 1 and 2). First, we conducted an analysis of a complete protein sequence data set representing all 1,284 SCOs shared by all taxa, including five distinct geographic isolates of T. pseudospiralis, and two outgroup species (that is, Trichuris suis and A. suum) using Bayesian inference, maximum likelihood and maximum parsimony to establish the phylogenetic relationships of all encapsulated and non-encapsulated Trichinella taxa. Here all three trees constructed had the same topology, with strong support values $(1.0 ; 87-100 \%)$ at all nodes, irrespective of the algorithm used (Fig. 1a). Second, employing the same algorithms, we independently assessed the relationships of all 16 Trichinella gene sets (without an outgroup) using protein sequences encoded by 2,855 SCOs shared by all taxa. Again, all three resultant trees had the same topology, consistently achieving very strong nodal support $(1.0 ; 94-100 \%)$, with the exception of the pair T. spiralis + T. nelsoni in the maximum parsimony analysis (support: 64\%). Third, to address this discrepancy, we studied separately the relationships of all encapsulated taxa using protein sequences 
Table 1 | Assembly and gene prediction statistics for the draft genomes of all recognized encapsulated Trichinella taxa`.

\begin{tabular}{|c|c|c|c|c|c|c|c|c|c|}
\hline Description & $\begin{array}{c}\text { T1 } \\
\text { ISS3 }\end{array}$ & $\begin{array}{c}\text { T2 } \\
\text { ISS10 }\end{array}$ & $\begin{array}{c}\text { T3 } \\
\text { ISS120 }\end{array}$ & $\begin{array}{c}\text { T5 } \\
\text { ISS417 }\end{array}$ & $\begin{array}{c}\text { T6 } \\
\text { ISS34 }\end{array}$ & $\begin{array}{c}\text { T7 } \\
\text { ISS37 }\end{array}$ & $\begin{array}{c}\text { T8 } \\
\text { ISS272 }\end{array}$ & $\begin{array}{c}\text { T9 } \\
\text { ISS409 }\end{array}$ & $\begin{array}{c}\text { T12 } \\
\text { ISS2496 }\end{array}$ \\
\hline Country of origin & Poland & Norway & Italy & USA & USA & Tanzania & Namibia & Japan & Argentina \\
\hline Host of origin & Domestic pig & Polar bear & Red fox & Coyote & Grizzly bear & Warthog & Lion & Raccoon dog & Cougar \\
\hline Genome size (bp) & $50,035,721$ & $48,088,508$ & $51,516,808$ & $49,039,267$ & $50,855,377$ & $47,453,436$ & $49,332,017$ & $49,096,069$ & $49,773,209$ \\
\hline Number of scaffolds; contigs & 7,$667 ; 9,209$ & 4,$635 ; 5,353$ & 8,$025 ; 9,709$ & 5,$255 ; 6,430$ & 5,$957 ; 7,471$ & 2,$973 ; 4,254$ & 4,$125 ; 5,876$ & 5,$824 ; 7,488$ & 6,$623 ; 8,003$ \\
\hline N50 (bp) & 212,546 & 141,195 & 147,150 & 106,482 & 158,103 & 293,867 & 239,129 & 212,690 & 154,103 \\
\hline N90 (bp) & 36,812 & 30,368 & 9,232 & 15,500 & 17,717 & 70,070 & 46,127 & 43,251 & 23,618 \\
\hline Genome GC content (\%) & 33.62 & 33.56 & 33.72 & 33.58 & 33.44 & 33.49 & 33.57 & 33.48 & 33.62 \\
\hline Coding GC content (\%) & 43.08 & 43.14 & 43.31 & 43.14 & 43 & 43.1 & 43.15 & 43.06 & 43.24 \\
\hline $\begin{array}{l}\text { Exonic proportion; including } \\
\text { introns (\%) }\end{array}$ & $33.08 ; 71.63$ & $33.71 ; 71.36$ & $34.09 ; 71.23$ & $33.62 ; 69.28$ & $33.54 ; 69.81$ & $33.87 ; 70.85$ & $33.31 ; 69.28$ & $35.26 ; 74.88$ & $35.88 ; 72.27$ \\
\hline $\begin{array}{l}\text { Number of putative coding } \\
\text { genes }\end{array}$ & 14,745 & 13,662 & 16,067 & 14,863 & 15,242 & 13,232 & 14,920 & 13,127 & 15,319 \\
\hline Mean gene size (bp) & 2,526 & 2,604 & 2,381 & 2,370 & 2,420 & 2,633 & 2,377 & 2,947 & 2,449 \\
\hline Mean CDS length (bp) & 1,045 & 1,093 & 962 & 1,017 & 1,003 & 1,108 & 1,026 & 1,061 & 965 \\
\hline Mean exon count per gene & 6.31 & 6.39 & 5.7 & 6.01 & 5.93 & 6.48 & 6.01 & 6.51 & 5.91 \\
\hline Mean exon length (bp) & 186.2 & 193.9 & 202.09 & 193.32 & 198.14 & 196.76 & 192.52 & 216.2 & 209.06 \\
\hline Mean intron length (bp) & 254.67 & 253.47 & 261.63 & 241.76 & 253.31 & 248.69 & 244.35 & 279.96 & 247.89 \\
\hline $\begin{array}{l}\text { Total length of coding } \\
\text { sequences }\end{array}$ & $27,499,002$ & $25,039,143$ & $29,213,671$ & $25,397,919$ & $26,529,424$ & $25,001,000$ & $25,015,560$ & $29,155,076$ & $25,830,832$ \\
\hline Repetitive sequences (\%) & 18.97 & 17.79 & 21.8 & 18.78 & 20.29 & 17.26 & 18.68 & 6.74 & 19.64 \\
\hline $\begin{array}{l}\text { CEG completeness: } \\
\text { complete; partial (\%) }\end{array}$ & $95.97 ; 97.18$ & $95.97 ; 97.18$ & $96.77 ; 97.18$ & $95.97 ; 97.18$ & $96.37 ; 97.18$ & $96.37 ; 97.18$ & $96.77 ; 97.18$ & $95.56 ; 97.18$ & $96.37 ; 97.18$ \\
\hline
\end{tabular}

Table 2 | Assembly and gene prediction statistics for the draft genomes of all recognized non-encapsulated Trichinella taxa`.

\begin{tabular}{|c|c|c|c|c|c|c|c|}
\hline Country of origin & Russia & Russia & Kazakhstan & USA & Australia & Thailand & Zimbabwe \\
\hline Host of origin & Raccoon & Brown rat & Tawny eagle & Black vulture & Spotted quoll & Human & Nile crocodile \\
\hline Number of scaffolds; contigs & 7,$287 ; 8,136$ & 7,$547 ; 7,647$ & 6,$600 ; 7,483$ & 6,$287 ; 7,079$ & 1,$381 ; 2,571$ & 2,$552 ; 3,122$ & 11,$275 ; 12,675$ \\
\hline N50 (bp) & 235,426 & 112,255 & 287,133 & 234,172 & 167,180 & 222,396 & 205,645 \\
\hline N90 (bp) & 60,266 & 9,250 & 69,797 & 50,288 & 50,779 & 64,723 & 8,776 \\
\hline Exonic proportion; including introns (\%) & $33.63 ; 72.68$ & $34.73 ; 71.61$ & $34.24 ; 73.39$ & $29.84 ; 61.96$ & $33.73 ; 67.98$ & $35.96 ; 76.49$ & $33.92 ; 72.45$ \\
\hline Number of putative coding genes & 12,699 & 13,754 & 12,462 & 14,708 & 11,006 & 11,854 & 14,933 \\
\hline Mean gene size (bp) & 2,955 & 2,620 & 3,053 & 2,071 & 2,944 & 3,169 & 2,591 \\
\hline Mean CDS length (bp) & 1,041 & 1,006 & 1,052 & 994 & 1,122 & 1,133 & 933 \\
\hline Mean exon count per gene & 6.58 & 6.22 & 6.66 & 5.91 & 6.64 & 6.92 & 5.87 \\
\hline Mean exon length (bp) & 210.71 & 207.52 & 217.94 & 169.78 & 222.84 & 217.53 & 209.41 \\
\hline
\end{tabular}

CDS, coding DNA sequence; CEG, core essential gene; ISS, Istituto Superiore di Sanità.

International Trichinella Reference Center (http://www.iss.it/site/Trichinella/) ISS codes are indicated.

${ }^{\star} \mathrm{T} 4=T$. pseudospiralis (including five distinct populations: $\mathrm{T} 4.1-\mathrm{T} 4.5$ ); $\mathrm{T} 10=T$. papuae; $\mathrm{T} 11=T$. zimbabwensis

encoded by 4,090 SCOs, and achieved strong support at all nodes for the three algorithms $(1.0 ; 99-100 \%)$. Then, to assess the root relationship of sister species $T$. spiralis $+T$. nelsoni to nonencapsulated clades, we employed the same approach to resolve the relationships of three encapsulated (T. spiralis, T. nelsoni and T. patagoniensis) and one non-encapsulated taxon (T. papuae) using protein sequences encoded by 4,300 SCOs, and achieved the same outcome (that is, absolute support at all nodes). The final consensus tree constructed (Fig. 1a) represents the results from the first and second analyses (for all three algorithms). As expected, the dendrogram constructed from syntenic correlation values (Supplementary Data 2) was consistent in topology with this consensus tree and supported the encapsulated and non-encapsulated clades (Fig. 1a).

For the encapsulated taxa, the consensus tree shows that a lineage with $T$. spiralis $+T$. nelsoni is the sister to other taxa, which hierarchically include $T$. patagoniensis, and pairs of sister species (T. nativa $+\mathrm{T} 6, T$. murrelli $+\mathrm{T} 9$ and T. britovi $+\mathrm{T} 8$; Fig. 1a). These relationships differ from those inferred in previous studies using few ribosomal or mitochondrial DNA sequences ${ }^{10,14,15}$, although some previously recognized sister species associations are concordant, and T. spiralis $+T$. nelsoni 
a

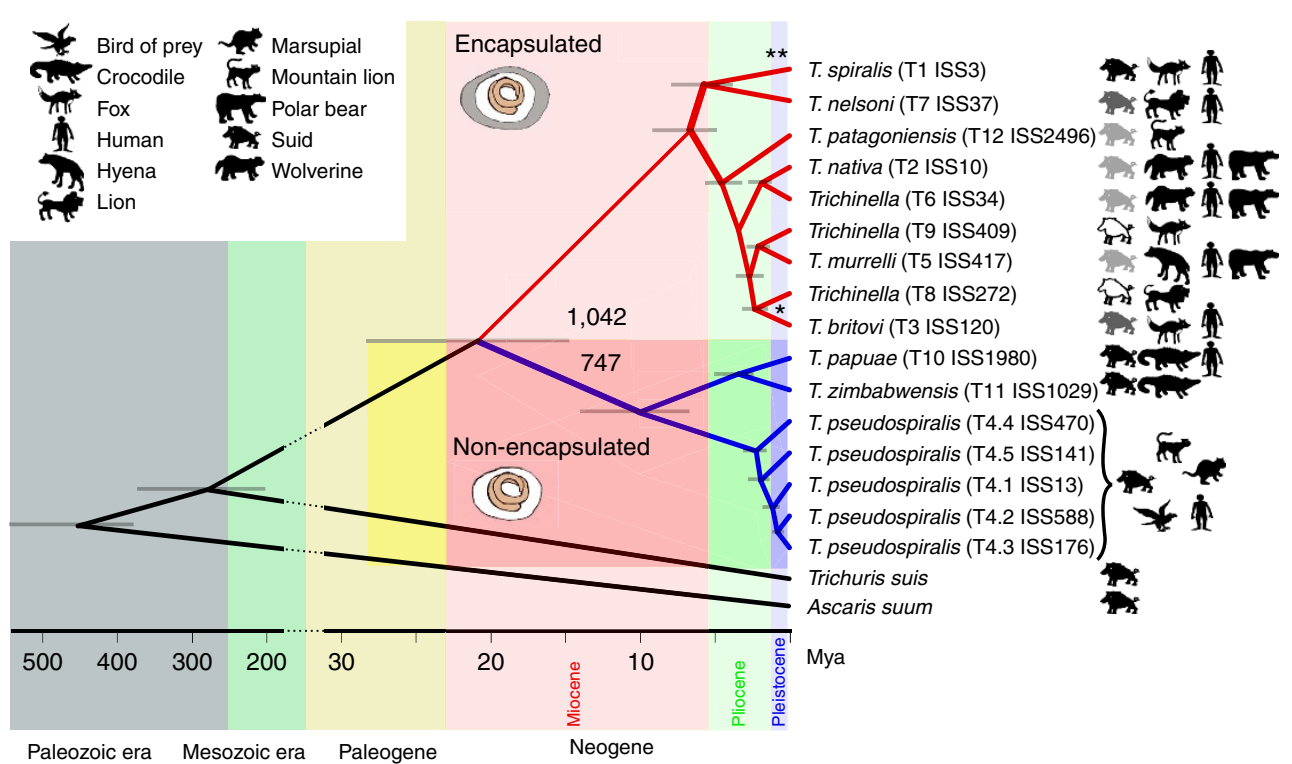

b

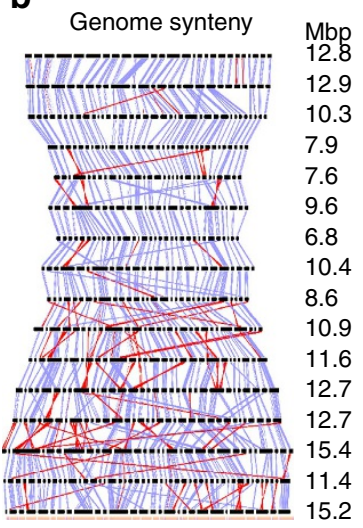

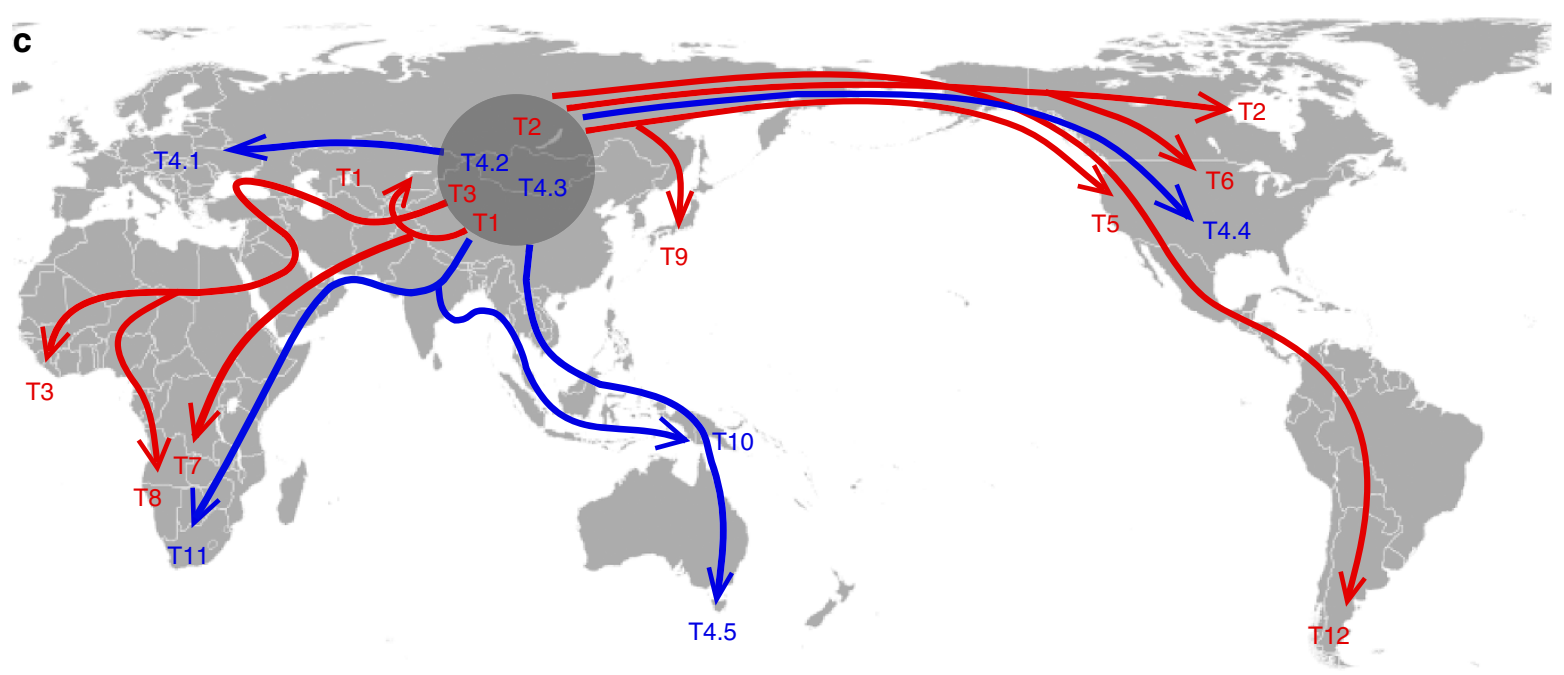

Figure 1 | The evolution and biogeography of Trichinella taxa. (a) The phylogeny of all 12 currently recognized taxa of Trichinella based on analyses of amino-acid sequence data from shared SCOs $(n=1,284)$ employing Bayesian infererence, ML and MP methods, with Trichuris suis and A. suum as outgroups; 1,042 and 747 are the numbers of orthologous gene groups, which are unique to encapsulated (red) and non-encapsulated (blue) Trichinella taxa, respectively. The topology of the trees constructed using each of these methods was the same; all nodes have absolute statistical support (1.00 or $100 \%$ ), except for one node $\left(^{\star}\right)$ in the ML analysis, where it was $99 \%$. The grey bars on the nodes represent $95 \%$ confidence intervals for the estimate of species branching time. T. spiralis (ISS195) ${ }^{19}$ shares the same phylogenetic position $\left(^{\star \star}\right)$ as T. spiralis (ISS3). Host animals: suids (Sus scrofa) represent both the sylvatic and domestic porcine hosts (left); the reproductive potential of a particular Trichinella taxon in S. scrofa ${ }^{12}$ is indicated by the colour scale: white: not assessed; light grey: low; dark grey: medium; black: high. Other animals (right) represent examples of carnivorous sylvatic hosts in different geographic regions, including fox, lion, mountain lion, marsupial, crocodile and bird of prey, and the accidental human host. (b) Representation of genome-wide synteny among Trichinella taxa (same order as listed on the right in a). Genomic scaffolds (black) sharing at least 10 SCOs between Trichinella taxa are displayed. A purple line indicates a single SCO and a red line an inverted SCO. The numbers on the right indicate the genomic length in megabases (Mb).

(c) Biogeography of Trichinella taxa proposed on the basis of known global (climate, extinctions and/or tectonic) events and diversification times (mya) for Trichinella taxa, estimated using a molecular clock approach. Encapsulated taxa: T. spiralis, T1; T. nativa, T2; T. britovi, T3; T. murrelli, T5; T. nelsoni, T7; T. patagoniensis, T12; and Trichinella genotypes T6, T8 and T9. Non-encapsulated taxa (infecting mammals, reptiles and/or birds): T. pseudospiralis, T4; T. papuae, T10; T. zimbabwensis, T11 (ref. 12). Geographic distributions of Trichinella taxa were reported by Pozio and Zarlenga ${ }^{11}$. The embedded public domain world map image (https://commons.wikimedia.org/wiki/File:BlankMap-World6.svg) has been modified using the programs World map tool v.1.16 (http://law.nagoya-u.ac.jp/en/appendix/software/worldmap) and GIMP v.2.8 (https://www.gimp.org).

and T. patagoniensis are relatively basal. At the time of initial phylogenetic assessment of Trichinella ${ }^{14}$, sequence data were not available for all five T. pseudospiralis isolates (T4.1-T4.5) or T. patagoniensis. Here, for non-encapsulated taxa, the pair T. papuae $+T$. zimbabwensis is basal to all representatives of T. pseudospiralis investigated (that is, T4.1-T4.5); T4.4 from
North America is basal to T4.5 from Tasmania and to all three isolates from Eurasia (T4.1-T4.3; Fig. 1a). Using the amino-acid sequences encoded by shared SCOs $(n=2,855)$, tree topology and nodal support values were similar to those achieved using wholemitochondrial data sets ${ }^{21}$, although these values were consistently high compared with previous analyses using small gene sets ${ }^{14,15}$. 
Here, for non-encapsulated taxa, the pair T. papuae+ T. zimbabwensis is basal to all representatives of T. pseudospiralis investigated (that is, T4.1-T4.5); T4.4 from North America is basal to T4.5 from Tasmania and to all three isolates from Eurasia (T4.1-T4.3; Fig. 1a). Therefore, we have now been able to establish, for the first time, the phylogenetic relationships of all currently recognized Trichinella taxa, including five $T$. pseudospiralis representatives (that is, T4.1-T4.5) and the more recently discovered T. patagoniensis.

Divergence and biogeography. Divergence time analysis based on the nematode diversification estimate of 532-382 million years ago (mya) $)^{33}$ (1,000 SCOs) implied that Trichinella and Trichuris suis had a most recent common ancestor (MRCA) 281 mya (95\% credible interval: 384-204), and that the encapsulated and non-encapsulated Trichinella taxa shared an MRCA $21(28-15)$ mya (Fig. 1a), coinciding with the transition from Oligocene to Miocene $^{34}$. Relative to a deep age for the Trichinella lineage, origin of a specific adaptation for encapsulation, associated with the radiation of nine taxa, occurred late in the evolutionary history of these nematodes. Subsequent diversification leading to extant species or species groups within the encapsulated and nonencapsulated clades is temporally circumscribed in the upper and uppermost Miocene during the Tortonian and Messinian periods $^{35,36}$, commencing $\sim 7(9-5)$ and 10 (14-7) mya (Fig. 1a) and continuing into the Pliocene and Pleistocene ${ }^{34}$. Despite major methodological differences, our estimates are very consistent with those of Zarlenga et al. ${ }^{14}$ in postulating a geographic distribution for the MRCA of all Trichinella taxa in Eurasia. Further concordance is seen in estimates for initial divergence of respective clades for encapsulated and nonencapsulated forms during the mid-Miocene, coincidental with perturbations in temperate ecosystems before diversification of extant species (Fig. 1a,c); this scenario might relate to an early Miocene glaciation $^{37}$, with low sea levels ${ }^{38}$ allowing a regional interchange of faunas linking Africa, Eurasia and North America $^{39}$. The diversification of non-encapsulated T. pseudospiralis from the common ancestor of T. papuae and T. zimbabwensis (in poikilotherms; Fig. 1a,c) coincides with the Tibetan plateau uplift and climate change around $10-8$ mya $^{40}$ and the divergence between $T$. papuae and T. zimbabwensis (4.9-2.3 mya), possibly contemporaneously with climate change and the Plio-Pleistocene extinction of some crocodylomorph reptiles $^{41}$. An avian host might explain the occurrence of T. pseudospiralis south of the Tibetan plateau.

Radiation of encapsulated Trichinella involves Eurasia, Africa, North America and South America. The occurrence of T. nelsoni (7.8-4.1 mya) and T. britovi $+\mathrm{T} 8$ (3.2-1.7 mya) on the African continent (Fig. 1c) follows temporally discrete and independent expansion events around 7.5 mya (Miocene), 4.5-4 and 3.5 mya (Pliocene) and 2.0 mya (Pleistocene) ${ }^{42}$. A separation of $T$. nelsoni $+T$. spiralis near the Miocene-Pliocene boundary further establishes the basis for an independent association with hominins and humans based on ecology and later domestication of primary suid hosts for the latter species ${ }^{43}$. We hypothesize that the diversification of $T$. britovi and T8 took place in Africa (Fig. 1c) as a consequence of biogeographic barriers and changing environmental conditions, which is in accord with a previous suggestion by Pozio et al. ${ }^{44}$ Alternatively, the isolation and divergence of an ancestral population across Eurasia and Africa, leading to T. britovi and T8, with later secondary expansion, would account for the extensive geographic range of the former species $^{10,14}$. We suggest that the loss of forests, formation of grasslands and the food scarcity for herbivores during the Miocene $\mathrm{M}^{45,46}$ across Eurasia and Africa resulted in a massive expansion of an entirely new guild of predators/hunters that were able to follow scarce prey over vast distances in the later Miocene, Pliocene and Pleistocene ${ }^{47}$. Encapsulated Trichinella taxa initially expanded into North America across Beringia during a time frame deeper than 5 mya, when the landmass was a permanent geographic feature linking Eurasia and the Nearctic, and before the inception of Northern Hemisphere glaciations ${ }^{48}$. The distribution of $T$. patagoniensis is consistent with the initial emergence of the Panamanian Isthmus $(\sim 10$ mya), as recently established $^{49-51}$. New chronologies are also consistent with the complex nature of faunal assembly in the Neotropical region, providing a mechanism for geographic colonization by small cats, followed by extensive radiation in South America after 8 mya, before the Great American Interchange ${ }^{52}$. Interestingly, there is no current evidence for geographic colonization of South America after 3 mya by nearctic species of Trichinella (for example, T. murrelli or T6), coincidental with large felids, procyonids, canids or mustelids (Fig. 1c). Inception of Northern Hemisphere glaciation cycles and periodic emergence of the Bering land bridge after 2.5 and 2.0 mya $^{53}$ led to independent episodes of geographic colonization and host-switching, driving patterns of isolation and genetic divergence (radiation) of Trichinella, linking Eurasia and the Nearctic ${ }^{48}$. Diversification of Eurasian/Nearctic sister species, including T. nativa $+\mathrm{T} 6$, T. murrelli + T9 and T. pseudospiralis in Northern America, reflects an intricate history in response to climate variation and habitat change, facilitating independent events of biotic expansion among carnivoran and other mammalian assemblages, including ursids, canids and mustelids primarily from Eurasia into North America (Fig. 1c). In addition, we hypothesize that T9 diverged from a common ancestor with T. murrelli before geographic colonization of the New World, or following isolation across Bering Strait during a glacial maximum (Fig. 1c). In the absence of a fossil record for Trichinella, it would be useful to determine the sequence of at least one Trichinella taxon from an extinct, infected vertebrate (for example, carnivoran) as a reference in time to assist future studies. The chronological and spatial history of Trichinella is described by episodic or cyclical patterns of independent geographic and host colonization on global and regional scales, the extensive development of mosaic faunal assemblages consistent with an integrated history for taxon pulses and ecological fitting mediated by climate and habitat variation over the late Tertiary $48,54,55$.

Parasite-host adaptation. The significantly higher GC content in the genomes and coding regions of encapsulated compared with non-encapsulated taxa (Kolmogorov-Smirnov (KS) test: $P$ values $=3.801 \times 10^{-4}$ and $7.466 \times 10^{-4}$, respectively; effect size $=1$ ) suggests that these two Trichinella clades may have adapted differently to varying environmental stresses (for example, temperature), host immune attacks and/or body temperatures (reptiles versus birds and mammals). We also propose that the intracellular lifestyle and associated bottleneck have resulted, throughout evolution, in a considerable reduction of genome size $(49 \mathrm{Mb})$ and lower GC content (33\%) in both encapsulated and non-encapsulated Trichinella taxa compared with extracellular relatives such as Trichuris suis $(78.5 \mathrm{Mb} ; 44 \%)^{22}$, which accords with observations for selected pathogens ${ }^{56,57}$.

Considering the morphological differences between encapsulated and non-encapsulated Trichinella taxa in host muscle cells ${ }^{12}$, we focused on exploring ES molecules likely to be associated with parasite-host interactions or host cell modification. Although more than half (52.9\%) of genes encoding putative ES proteins could not be annotated (Supplementary Data 1), we identified seven SCOs (groups (GRPs) 2,426, 4,153, 
2,136, 3,720, 2,832, 3,607 and 2,897) encoding ES orphans whose gene transcription is significantly upregulated exclusively in encapsulated taxa, and which are hypothesized to modulate host immune responses or immune evasion. By contrast, we identified an SCO (GRP 2254) encoding a cathelidicin-like molecule whose transcription is upregulated only in all non-encapsulated taxa, and might suppress antigen processing and/or presentation, a proposal supported by some evidence for an orthologue (FhHDM-1) in the parasitic flatworm Fasciola hepatica ${ }^{58}$. In both encapsulated and non-encapsulated taxa, we identified an orphan protein (GRP 838; GenBank accession: BG520575) that possesses a structural motif that is consistent with mitochondrial cytochrome $c$ (Supplementary Data 3 ) and/or the mitochondrial dynamics protein of $51 \mathrm{kDa}$ (MID-51) ${ }^{59}$ detected previously in $T$. spiralis $^{30,60}$. This ES protein likely modifies host cells by uncoupling the mitochondria to maintain an anaerobic state required by the first larval stage (L1) of Trichinella ${ }^{61,62}$ (Supplementary Data 3). Homologues of the $45-\mathrm{kDa}$ secreted proteins $^{30,32}$ (GeneBank accession: AAA20539.1) were encoded in all Trichinella taxa (GRP 70; Supplementary Data 3); the predicted structures of these proteins match plasminogens and thrombins (Supplementary Data 3), which are serine proteases with roles in pro-collagen production and/or the activation of collagenases $^{63,64}$, respectively.

Subsequently, we identified 1,042 orthologous gene groups (GRPs) exclusively in genomes of the encapsulated taxa, and 747 were exclusive to non-encapsulated taxa (Fig. 1a). We explored the distinctiveness between all encapsulated and non-encapsulated taxa, for which RNA sequencing (RNA-seq) data were available by comparing their transcription profiles at the L1 stage for all SCOs $(n=2,855$; Supplementary Tables 3 and 4$)$. This analysis identified SCOs that were uniquely transcribed in encapsulated $(n=47)$ and non-encapsulated $(n=68)$ taxa (Supplementary Data 3 ). Interestingly, the average GC content of all of these 115 SCOs is significantly lower (KS test: $P$ value $<3.059 \times 10^{-7}$; effect size $\left.=1\right)$ than that of all genes of all Trichinella taxa. In addition, the significantly lower AT content of differentially transcribed genes (KS test: $P$ value $=4.076 \times 10^{-2}$; effect size $\left.=0.67\right)$ in non-encapsulated taxa suggests a distinct adaptation of genes to the muscle cell compared with encapsulated taxa.

Interestingly, we found expansions in two orthologous groups in non-encapsulated taxa. The first (GRP 149) represents a serine protease precursor with two trypsin-like domains (TsSerP and AF331156) ${ }^{65}$, which is expanded in all non-encapsulated taxa (Supplementary Data 3 ) and appears to play a vital role in larval feeding and/or moulting ${ }^{65}$. The second group represents a multicystatin-like domain protein (MCD-1) ${ }^{32}$ (GRP 482) encoded by six gene copies in T. papuae, and two copies in T. pseudospiralis (T4.5) from Australia and T. zimbabwensis compared with one copy in all other Trichinella taxa. This protein contains three repeated domains, each with similarity to family 2 cystatins $^{66}$, but lacking critical consensus sites for cysteine protease inhibition. In this respect, the domain organization is similar to that of mammalian kininogens and a known six-domain cystatin from $F$. hepatica ${ }^{67}$. We propose that MCD-1 might function as a cytokine antagonist by binding to transforming growth factors (TGF)- $\beta 1$ and $-\beta 2$ in a manner similar to fetuin 68 , a proposal that is consistent with previous findings for $T$. spiralis and evidence of low-level expression of TGF- $\beta$ in the epithelium of the jejunum of infected mice ${ }^{69}$. Certainly, family 2 cystatins secreted by other parasitic nematodes have known roles in immune evasion, including the inhibition of proteases involved in antigen presentation and modulation of cytokine responses $^{70}$. Although MCD-1 is unlikely to function as a typical cystatin, it is expressed at the parasite-host interface ${ }^{32}$ and might modulate host immune responses or enable immune evasion. An expanded set of MCD-1s might allow some non-encapsulated Trichinella taxa to suppress immune responses better than encapsulated taxa, possibly facilitating dissemination into a wider range of vertebrate hosts ${ }^{12}$.

\section{Discussion}

Using Illumina-based sequencing and bioinformatics, we assembled draft nuclear genomes representing all 12 recognized taxa of Trichinella, including five geographic isolates of T. pseudospiralis. Using extensive amino-acid sequence data sets derived from all shared SCOs from these nuclear genomes and/or outgroup taxa, we were able to reconstruct the phylogeny of Trichinella. In a previous study, Zarlenga et al. ${ }^{14}$ had proposed the phylogenetic relationships of Trichinella species and genotypes using small DNA sequence data sets. At the time, these authors provided comprehensive interpretations of the findings and concluded that post-Miocene expansion, colonization and host-switching drove speciation among extant members of the genus Trichinella; although their study was very informative and interesting, the resolution of some clades and the positions of some of the taxa, such as T. nativa and T6 as well as T. murrelli and T9, did not always appear to be well supported statistically by the data presented, when outgroups (mermithids and Trichuris spp.) were included in the analyses. The reason for the limited resolution of some relationships was likely because of the use of sequence data set representing only selected genetic loci (nuclear small subunit and second internal transcribed spacer; mitochondrial large subunit and cytochrome $c$ oxidase subunit $1)^{14}$. In addition, it appears that some of the species selected as outgroups might have been too distant to achieve a robust phylogeny using all of the concatenated sequence data in the analyses.

Here we utilized an extensive amino-acid sequence data set representing all SCOs (concatenated alignment over 597,495 amino-acid positions) originating from all 16 genomes representing all members of the Trichinella complex, including all known geographic isolates of T. pseudospiralis and T. patagoniensis, not previously available 14,15 , as well as Trichuris suis and A. suum (outgroups) in the analyses. By contrast, in a previous study ${ }^{21}$ we could not use Trichuris or Ascaris as outgroups because of excessive sequence divergence in predicted mitochondrial proteins (for example, NAD4 and NAD6) encoded by some genes, such that an unambiguous alignment of homologous characters was impossible; these outgroups therefore had to be excluded from the analyses of mitochondrial data sets, as their inclusion would have led to erroneous results and interpretation. In the present study, the definition of 1,284-2,855 SCOs among all Trichinella taxa (with or without outgroups) allowed, employing an iterative, stepwise approach, the reconstruction of a robust phylogeny utilizing three independent tree-building methods. In accordance with previous investigations ${ }^{14,21,68,69}$, the present analyses showed that the encapsulated taxa grouped separately from non-encapsulated taxa. Importantly, we were also able to resolve, for the first time, the phylogenetic positions of T. spiralis $+T$. nelsoni and T. murrelli $+\mathrm{T} 9$, five representatives of T. pseudospiralis and T. patagoniensis in relation to all other taxa, with strong (99-100\%) statistical support. The diversification times of Trichinella matched well with known historical global events and allowed the biogeography of all taxa to be reconstructed. This biogeography clearly supports the notion that encapsulated and non-encapsulated taxa frequently occur in sympatry and in the context of faunal assembly, driven strongly by events of geographic and host colonization, involving complex spatial and chronological mosaics linking evolutionary 
and ecological time ${ }^{54}$. Nonetheless, in the future, extensive sampling of Trichinella taxa from around the world will be required to explore, in depth, population genetic structures to reassess the present phylogenic and biogeographic reconstruction, and to attempt to identify the host origin of ancestral forms of T. spiralis, the principal causative agent of human trichinellosis. In conclusion, although the present study focused sharply on fundamental phylogenetic and biogeographic aspects, the genomic and transcriptomic resources created here will substantially accelerate many fundamental and applied areas of Trichinella/trichinellosis research. In addition, the genome-wide approach that we employed should have applicability to a wide range of parasites and other metazoan organisms.

\section{Methods}

Production and procurement of Trichinella taxa. Sixteen samples (isolates) of first-stage larvae (L1s) representing all 12 recognized species and genotypes of Trichinella were produced at the International Trichinella Reference Center (http://www.iss.it/site/Trichinella/), Istituto Superiore di Sanità (ISS), Rome, Italy. The 16 samples included the following: one sample of each T. spiralis (code: ISS3), T. nativa (ISS10), T. britovi (ISS120), T. murrelli (ISS417), T. nelsoni (ISS37), T. patagoniensis (ISS2496) as well as Trichinella genotypes T6 (ISS34), T8 (ISS272) and T9 (ISS409); five samples representing distinct geographic isolates of T. pseudospiralis (ISS13, ISS588, ISS176, ISS470 and ISS141 = T4.1, T4.2, T4.3, T4.4 and T4.5, respectively); and one sample of each T. papuae (ISS1980) and T. zimbabwensis (ISS1029; cf. Tables 1 and 2). These samples were individually produced in female CD1 mice. L1s were recovered from host muscles by pepsin (1\%)- $\mathrm{HCl}(1 \%)$ digestion at $40{ }^{\circ} \mathrm{C}$ for $30 \mathrm{~min}$, sedimented, washed extensively in physiological saline and suspended in $90 \%$ ethanol for storage $\mathrm{e}^{71}$. Each sample, which represented a packed volume of $50 \mu \mathrm{l}$ of L1s, was snap-frozen in liquid nitrogen and kept at $-80^{\circ} \mathrm{C}$ until nucleic acid isolation.

RNA-seq and transcriptome assembly. Total RNA was isolated separately from L1s of each isolate of Trichinella employing the TriPure isolation reagent (Roche Molecular Biochemicals). Packed volumes of 20-50 $\mu$ l were used, equating to thousands of larvae. RNA yields were estimated spectrophotometrically (NanoDrop 1000), and the integrity of RNA was verified using the BioAnalyzer. RNA-seq was conducted using an established $\operatorname{method}^{72}$ and relevant data summarized ( $c f$. Supplementary Tables 3 and 4). De novo assemblies were performed using a software pipeline, incorporating the programme Trimmomatic v.0.27 (http://www.usadellab.org/cms/?page=trimmomatic) for read qualityfiltering, Khmer v.1.1 (ref. 73) for the reduction of high and low coverage reads, and Velvet v.1.2.07 (ref. 74) and Oases v.0.2.08 (ref. 75) for sequence assembly.

Other methods. Genome sequencing and assembly, prediction of repetitive elements, prediction of protein-encoding genes, functional annotation, phylogenetic and divergence time analyses, synteny, GC content and differential transcription analyses are described in Supplementary Methods.

\section{References}

1. Pozio, E. World distribution of Trichinella spp. infections in animals and humans. Vet. Parasitol. 149, 3-21 (2007).

2. Murrell, K. D. \& Pozio, E. Worldwide occurrence and impact of human trichinellosis, 1986-2009. Emerg. Infect. Dis. 17, 2194-2202 (2011).

3. Owen, I. L., Gomez Morales, M. A., Pezzotti, P. \& Pozio, E. Trichinella infection in a hunting population of Papua New Guinea suggests an ancient relationship between Trichinella and human beings. Trans. R Soc. Trop. Med. Hyg. 99, 618-624 (2005).

4. Ribicich, M., Gamble, H. R., Rosa, A., Bolpe, J. \& Franco, A. Trichinellosis in Argentina: an historical review. Vet. Parasitol. 132, 137-142 (2005).

5. Blaga, R. et al. A dramatic increase in the incidence of human trichinellosis in Romania over the past 25 years: impact of political changes and regional food habits. Am. J. Trop. Med. Hyg. 76, 983-986 (2007).

6. Barennes, H. et al. A major trichinellosis outbreak suggesting a high endemicity of Trichinella infection in northern Laos. Am. J. Trop. Med. Hyg. 78, 40-44 (2008).

7. Vu Thi, N. et al. High prevalence of anti-Trichinella IgG in domestic pigs of the Son La province, Vietnam. Vet. Parasitol. 168, 136-140 (2010).

8. Cui, J., Wang, Z. Q. \& Xu, B. L. The epidemiology of human trichinellosis in China during 2004-2009. Acta Trop. 118, 1-5 (2011).

9. Cuttell, L., Cookson, B., Jackson, L. A., Gray, C. \& Traub, R. J. First report of a Trichinella papuae infection in a wild pig (Sus scrofa) from an Australian island in the Torres Strait region. Vet. Parasitol. 185, 343-345 (2012).
10. Pozio, E., Hoberg, E., La Rosa, G. \& Zarlenga, D. S. Molecular taxonomy, phylogeny and biogeography of nematodes belonging to the Trichinella genus. Infect. Genet. Evol. 9, 606-616 (2009).

11. Pozio, E. \& Zarlenga, D. S. New pieces of the Trichinella puzzle. Int. J. Parasitol 43, 983-997 (2013).

12. Pozio, E. \& Murrell, K. D. Systematics and epidemiology of Trichinella. Adv. Parasitol. 63, 367-439 (2006)

13. Krivokapich, S. J. et al. Trichinella patagoniensis n. sp. (Nematoda), a new encapsulated species infecting carnivorous mammals in South America. Int. J. Parasitol. 42, 903-910 (2012).

14. Zarlenga, D. S., Rosenthal, B. M., La Rosa, G., Pozio, E. \& Hoberg, E. P. Post-Miocene expansion, colonization, and host switching drove speciation among extant nematodes of the archaic genus Trichinella. Proc. Natl Acad. Sci. USA 103, 7354-7359 (2006).

15. Krivokapich, S. J. et al. Molecular evidence for a novel encapsulated genotype of Trichinella from Patagonia, Argentina. Vet. Parasitol. 156, 234-240 (2008).

16. Mardis, E. R. The impact of next-generation sequencing technology on genetics. Trends Genet. 24, 133-141 (2008).

17. Cantacessi, C., Campbell, B. E. \& Gasser, R. B. Key strongylid nematodes of animals-impact of next-generation transcriptomics on systems biology and biotechnology. Biotechnol. Adv. 30, 469-488 (2012).

18. Koboldt, D. C., Steinberg, K. M., Larson, D. E., Wilson, R. K. \& Mardis, E. R The next-generation sequencing revolution and its impact on genomics. Cell 155, 27-38 (2013).

19. Mitreva, M. et al. The draft genome of the parasitic nematode Trichinella spiralis. Nat. Genet. 43, 228-235 (2011).

20. Webb, K. M. \& Rosenthal, B. M. Next-generation sequencing of the Trichinella murrelli mitochondrial genome allows comprehensive comparison of its divergence from the principal agent of human trichinellosis, Trichinella spiralis. Infect. Genet. Evol. 11, 116-123 (2011).

21. Mohandas, N. et al. Mitochondrial genomes of Trichinella species and genotypes-a basis for diagnosis, and systematic and epidemiological explorations. Int. J. Parasitol. 44, 1073-1080 (2014)

22. Jex, A. R. et al. Genome and transcriptome of the porcine whipworm Trichuris suis. Nat. Genet. 46, 701-706 (2014).

23. Jex, A. R. et al. Ascaris suum draft genome. Nature 479, 529-533 (2011).

24. Schwarz, E. M. et al. The genome and developmental transcriptome of the strongylid nematode Haemonchus contortus. Genome Biol. 14, R89 (2013).

25. Laing, R. et al. The genome and transcriptome of Haemonchus contortus, a key model parasite for drug and vaccine discovery. Genome Biol. 14, R88 (2013).

26. Zhu, X.-Q. et al. Genetic blueprint of the zoonotic pathogen Toxocara canis. Nat. Commun. 6, 6145 (2015).

27. Ghedin, E. et al. Draft genome of the filarial nematode parasite Brugia malayi. Science 317, 1756-1760 (2007).

28. C. elegans Sequencing Consortium, C. Genome sequence of the nematode $C$. elegans: a platform for investigating biology. Science 282, 2012-2018 (1998).

29. Robinson, M. W. \& Connolly, B. Proteomic analysis of the excretory-secretory proteins of the Trichinella spiralis L1 larva, a nematode parasite of skeletal muscle. Proteomics 5, 4525-4532 (2005).

30. Robinson, M. W., Gare, D. C. \& Connolly, B. Profiling excretory/secretory proteins of Trichinella spiralis muscle larvae by two-dimensional gel electrophoresis and mass spectrometry. Vet. Parasitol. 132, 37-41 (2005).

31. Robinson, M. W., Greig, R., Beattie, K. A., Lamont, D. J. \& Connolly, B. Comparative analysis of the excretory-secretory proteome of the muscle larva of Trichinella pseudospiralis and Trichinella spiralis. Int. J. Parasitol. 37, 139-148 (2007)

32. Robinson, M. W., Massie, D. H. \& Connolly, B. Secretion and processing of a novel multi-domain cystatin-like protein by intracellular stages of Trichinella spiralis. Mol. Biochem. Parasitol. 151, 9-17 (2007).

33. Rota-Stabelli, O., Daley, A. C. \& Pisani, D. Molecular timetrees reveal a Cambrian colonization of land and a new scenario for ecdysozoan evolution. Curr. Biol. 23, 392-398 (2013).

34. McGowran, B. et al. Neogene and quaternary coexisting in the geological time scale: the inclusive compromise. Earth Sci. Rev. 96, 249-262 (2009).

35. Hilgen, F. et al. The Global boundary Stratotype Section and Point (GSSP) of the Tortonian stage (Upper Miocene) at Monte Dei Corvi. Episodes 28, 6-17 (2005).

36. Hilgen, F. et al. The Global Boundary Stratotype Section and Point (GSSP) of the Messinian stage (uppermost Miocene). Episodes 23, 172-178 (2000).

37. Leckie, R. M. \& Webb, P.-N. Late oligocene-early miocene glacial record of the Ross Sea, Antarctica: evidence from DSDP Site 270. Geology 11, 578-582 (1983).

38. Keller, G. \& Barron, J. A. Paleoceanographic implications of Miocene deep-sea hiatuses. Geol. Soc. Am. Bull. 94, 590-613 (1983).

39. Janis, C. M. Tertiary mammal evolution in the context of changing climates, vegetation, and tectonic events. Annu. Rev. Ecol. Systematics 24, 467-500 (1993). 
40. Molnar, P., England, P. \& Martinod, J. Mantle dynamics, uplift of the Tibetan Plateau, and the Indian monsoon. Rev. Geophys. 31, 357-396 (1993).

41. Markwick, P. J. Crocodilian diversity in space and time: the role of climate in paleoecology and its implication for understanding K/T extinctions. Paleobiology 24, 470-497 (1998).

42. Werdelin, L. \& Peigne, S. in Cenozoic Mammals of Africa. (eds Werdelin, L. \& Sanders, W. J.) 1008 (University of California Press, 2010).

43. Rosenthal, B. M. et al. Human dispersal of Trichinella spiralis in domesticated pigs. Infect. Genet. Evol. 8, 799-805 (2008).

44. Pozio, E. et al. Trichinella britovi etiological agent of sylvatic trichinellosis in the Republic of Guinea (West Africa) and a re-evaluation of geographical distribution for encapsulated species in Africa. Int. J. Parasitol. 35, 955-960 (2005).

45. Retallack, G. J. Cenozoic expansion of grasslands and climatic cooling. J. Geol. 109, 407-426 (2001).

46. Keeley, J. E. \& Rundel, P. W. Fire and the Miocene expansion of C4 grasslands. Ecol. Lett. 8, 683-690 (2005).

47. Van Valkenburgh, B. Major patterns in the history of carnivorous mammals Annu. Rev. Earth Planet. Sci. 27, 463-493 (1999).

48. Hoberg, E. P., Galbreath, K. E., Cook, J. A., Kutz, S. J. \& Polley, L. Northern host-parasite assemblages: history and biogeography on the borderlands of episodic climate and environmental transition. Adv. Parasitol. 79, 1-97 (2012).

49. Bacon, C. D. et al. Biological evidence supports an early and complex emergence of the Isthmus of Panama. Proc. Natl Acad. Sci. USA 112, 6110-6115 (2015).

50. Hoorn, C. \& Flantua, S. Geology. An early start for the Panama land bridge. Science 348, 186-187 (2015).

51. Montes, C. et al. Middle Miocene closure of the Central American Seaway. Science 348, 226-229 (2015).

52. Johnson, W. E. et al. The late Miocene radiation of modern Felidae: a genetic assessment. Science 311, 73-77 (2006).

53. Ogasawara, K. Review and comments on late Neogene climatic fluctuations and the intermittence of the Bering Land Bridge. J. Asian Earth Sci. 16, 45-48 (1998).

54. Hoberg, E. P. \& Brooks, D. R. A macroevolutionary mosaic: episodic hostswitching, geographical colonization and diversification in complex hostparasite systems. J. Biogeogr. 35, 1533-1550 (2008).

55. Hoberg, E. P. \& Brooks, D. R. Evolution in action: climate change, biodiversity dynamics and emerging infectious disease. Philos. Trans. R Soc. Lond. B. Biol. Sci. 370, pii: 20130553. doi:10.1098/rstb.2013.0553 (2015).

56. Bastolla, U., Moya, A., Viguera, E. \& van Ham, R. C. Genomic determinants of protein folding thermodynamics in prokaryotic organisms. J. Mol. Biol. 343, 1451-1466 (2004).

57. Bastien, O. et al. Analysis of the compositional biases in Plasmodium falciparum genome and proteome using Arabidopsis thaliana as a reference. Gene 336, 163-173 (2004).

58. Robinson, M. W. et al. A helminth cathelicidin-like protein suppresses antigen processing and presentation in macrophages via inhibition of lysosomal vATPase. FASEB J. 26, 4614-4627 (2012).

59. Richter, V. et al. Structural and functional analysis of MiD51, a dynamin receptor required for mitochondrial fission. J. Cell Biol. 204, 477-486 (2014)

60. Mitreva, M. et al. Gene discovery in the adenophorean nematode Trichinella spiralis: an analysis of transcription from three life cycle stages. Mol. Biochem. Parasitol. 137, 277-291 (2004).

61. Capo, V. A., Despommier, D. D. \& Polvere, R. I. Trichinella spiralis: vascular endothelial growth factor is up-regulated within the nurse cell during the early phase of its formation. J. Parasitol. 84, 209-214 (1998).

62. Despommier, D. D. How Does trichinella spiralis make itself at home? Parasitol. Today 14, 318-323 (1998).

63. Matrisian, L. M. The matrix-degrading metalloproteinases. Bioessays 14, 455-463 (1992)

64. Chambers, R. C. et al. Thrombin stimulates fibroblast procollagen production via proteolytic activation of protease-activated receptor 1. Biochem. J. 333, 121-127 (1998)

65. Trap, C. et al. Cloning and analysis of a cDNA encoding a putative serine protease comprising two trypsin-like domains of Trichinella spiralis. Parasitol. Res. 98, 288-294 (2006).

66. Ochieng, J. \& Chaudhuri, G. Cystatin superfamily. J. Health Care Poor Underserved. 21, 51-70 (2010).

67. Khaznadji, E., Collins, P., Dalton, J. P., Bigot, Y. \& Moire, N. A new multidomain member of the cystatin superfamily expressed by Fasciola hepatica. Int. J. Parasitol. 35, 1115-1125 (2005).

68. Demetriou, M., Binkert, C., Sukhu, B., Tenenbaum, H. C. \& Dennis, J. W. Fetuin/alpha 2-HS glycoprotein is a transforming growth factor-beta type II receptor mimic and cytokine antagonist. J. Biol. Chem. 271, 12755-12761 (1996).
69. Knight, P. A. et al. Expression profiling reveals novel innate and inflammatory responses in the jejunal epithelial compartment during infection with Trichinella spiralis. Infect. Immun. 72, 6076-6086 (2004).

70. Hartmann, S. \& Lucius, R. Modulation of host immune responses by nematode cystatins. Int. J. Parasitol. 33, 1291-1302 (2003).

71. Pozio, E. \& La Rosa, G. Molecular Detection of Foodborne Pathogens (CRC Press, Taylor \& Francis Group, 2010).

72. Mortazavi, A. et al. Scaffolding a Caenorhabditis nematode genome with RNA-seq. Genome Res. 20, 1740-1747 (2010).

73. Brown, C. T., Howe, A., Zhang, Q., Pyrkosz, A. B. \& Brom, T. H. A reference free algorithm for computational normalization of shotgun sequencing data. http://arxiv.org/abs/1203.4802 (2012).

74. Zerbino, D. R. \& Birney, E. Velvet: algorithms for de novo short read assembly using de Bruijn graphs. Genome Res. 18, 821-829 (2008).

75. Schulz, M. H., Zerbino, D. R., Vingron, M. \& Birney, E. Oases: robust de novo RNA-seq assembly across the dynamic range of expression levels. Bioinformatics 28, 1086-1092 (2012).

\section{Acknowledgements}

Funding from the Australian Research Council, the National Health and Medical Research Council (NHMRC) of Australia, Yourgene Bioscience and Melbourne Water Corporation is gratefully acknowledged (R.B.G. et al.). This project was funded in part through DG SANCO of the European Commission and Istituto Superiore di Sanità, Rome (E.P.). This project was also supported by a Victorian Life Sciences Computation Initiative (grant number VR0007) on its Peak Computing Facility at the University of Melbourne, an initiative of the Victorian Government (R.B.G.). N.D.Y. holds an NHMRC Early Career Research Fellowship. P.W.S. thanks the Howard Hughes Medical Institute (HHMI) and the National Institutes of Health (NIH). We thank the staff of Yourgene Bioscience for their contributions. We would specifically like to acknowledge all scientists who developed the programmes used in this study. Given restrictions on the number of articles that could be cited, we were unable to include all original articles in the Methods section. Instead, we have included links to respective websites. P.K.K. is the recipient of a scholarship (STRAPA) from the University of Melbourne.

\section{Author contributions}

P.K.K., N.D.Y. and R.B.G. wrote the paper with inputs from all other co-authors including B.C.H.C., A.R.J., P.T. and P.R.B. G.L.R., E.P. and colleagues produced parasite material. G.L.R. prepared all nucleic acids and undertook laboratory-based evaluations. P.K.K. undertook the genome assembly and all bioinformatic analyses, with support from N.D.Y. and R.B.G. A.V.K. provided initial support with phylogeny. A.H. provided support with structural modelling. E.P.H. explored aspects of historical biogeography and faunal assembly. With support from G.L.R. and E.P., R.B.G. led the project.

\section{Additional information}

Accession codes: This Whole Genome Shotgun project has been deposited in the NCBI BioProject database with accession code PRJNA257433. The project includes: all the genome assemblies under accession codes JYDH00000000 to JYDW00000000; raw Illumina read sets for the genomic DNAs; raw reads for RNA-seq under accession codes SRS672137, SRS898754, SRS900949, SRS906989, SRS906991 to SRS906994, SRS906996, SRS906998 to SRS907002, SRS908054 and SRS908055; and assembled transcriptomes for the 12 Trichinella taxa under accession codes GEBM00000000 to GEBP00000000, GEBR00000000 to GEBZ00000000, GECA00000000 and GECJ00000000. The versions described in this paper are JYDH01000000 to JYDW01000000 for the genomes, and GEBM01000000 to GEBP01000000, GEBR01000000 to GEBZ01000000, GECA01000000 and GECJ01000000 for the transcriptomes.

Supplementary Information accompanies this paper at http://www.nature.com/ naturecommunications

Competing financial interests: The authors declare no competing financial interests.

Reprints and permission information is available online at http://npg.nature.com/ reprintsandpermissions/

How to cite this article: Korhonen, P. K. et al. Phylogenomic and biogeographic reconstruction of the Trichinella complex. Nat. Commun. 7:10513 doi: 10.1038/ncomms10513 (2016).

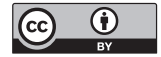

This work is licensed under a Creative Commons Attribution 4.0 International License. The images or other third party material in this article are included in the article's Creative Commons license, unless indicated otherwise in the credit line; if the material is not included under the Creative Commons license, users will need to obtain permission from the license holder to reproduce the material To view a copy of this license, visit http://creativecommons.org/licenses/by/4.0/ 